\title{
The Short Term Influence of RV Pacing Burden on Numerous Echocardiographic and Spiroergometric Parameter In Patients With Preserved LVEF - The Mechanism of The Worsened Clinical Outcome Due To High RV Pacing Burden Remains Unclear.
}

\section{Akram Youssef}

Technische Universität Dresden, Campus Chemnitz

Karim Ibrahim

Technische Universität Dresden: Technische Universitat Dresden

Michael Günther

Technische Universität Dresden: Technische Universitat Dresden

\section{Steffen Kolschmann}

Technische Universität Dresden: Technische Universitat Dresden

Utz Richter

Technische Universität Dresden: Technische Universitat Dresden

\section{Alexander Francke}

HELIOS Hospital Pirna: HELIOS Klinikum Pirna

Carsten Wunderlich

HELIOS Hospital Pirna: HELIOS Klinikum Pirna

Marian Christoph ( $\square$ marian.christoph@tu-dresden.de)

Klinikum Chemnitz-MEDIC https://orcid.org/0000-0003-4053-4202

\section{Research article}

Keywords: Ventricular pacing, Pacemaker, Echocardiography, Spiroergometry, Clinical outcome

Posted Date: June 29th, 2021

DOl: https://doi.org/10.21203/rs.3.rs-659020/v1

License: (1) (i) This work is licensed under a Creative Commons Attribution 4.0 International License. Read Full License 


\section{Abstract}

Background: The cause of worsened clinical outcome due to high RV pacing burden remains unclear.

Objective: To investigate the impact of RV pacing on several echocardiographic and spiroergometric parameter

Methods: In 60 pacemaker patients with preserved LVEF serial echocardiographies and spiroergometries were performed over a time course of 12 months. Additionally in 50 patients retrospective echocardiographic analyses of the LV- and RV function were carried out up to 24 months after pacemaker implantation.

Results: The patients were divided into two groups: The high RV pacing burden group (hRVP: $\geq 40 \%$ ) and the low RV pacing group (IRVP $<40 \%$ ) according to the definitions in previous randomized MOST and DAVID trials. After a period of 12 month pacemaker therapy there could not revealed any changes LVEDD, LVESD, LVEF, E/A-ratio; E/E'-ratio and TAPSE independently of the RV pacing burden. Additionally, after 24 month long term follow-up there were no changes in LVEF and TAPSE in both groups. Accordingly to these echo data no relevant changes of peak exercise capacity, ventilatory anaerobic threshold and maximal oxygen consumption could be revealed independently of the RV pacing.

Conclusions: In pacemaker patients with preserved LVEF the burden of RV pacing has no adverse influence, neither to several echocardiographic parameters nor to the clinical exercise capacity after a follow-up of 12 to 24 month. Therefore, the mechanism of the worsened clinical outcome due to high RV pacing burden in patients without a relevant structural heart disease remains unclear.

\section{Introduction}

The cause of worsened clinical outcome due to high RV pacing burden remains unclear. On the one hand some clinical studies suggest an adverse clinical effect of RV pacing. In this context, the DAVID trial and the MOST trial revealed increased rates of death or hospitalization for heart failure and increased incidence of atrial fibrillation in patients with a cumulative RV pacing $>40 \% .{ }^{1-3}$ On the other hand some long term registry data could not confirm changes of LVEF in dependence of the RV pacing burden. ${ }^{4}$ Therefore in the present clinical registry the influence of RV pacing burden on several echocardiographic parameter were evaluated. In addition, serial spiroergometric examinations were performed to investigate the clinically functional outcome of the pacemaker patients.

\section{Material And Methods Study Design}

The current study was a single-centre registry, divided into a prospective and a retrospective part, performed in compliance with the guidelines for good clinical practice and the Declaration of Helsinki. 
The study was approved by the institutional ethical review board. All data were collected, managed and analysed at the Heart Centre, University of Dresden (ethics votum University of Dresden: EK 28409202).

The primary endpoints of this study were the development of the left ventricular end diastolic and end systolic diameter of the left ventricle (LVEDD, LVESD), the systolic left ventricular function (LVEF), the diastolic left ventricular function (E/A-ratio; E/E'-ratio) and the systolic right ventricular function (TAPSE) in dependence of the RV apical pacing burden.

The primary clinical endpoints were the development of maximal oxygen consumption $\left(\mathrm{VO}_{2} \mathrm{max}\right)$, maximal workload and ventilatory anaerobic threshold (VAT) in dependence of the RV apical pacing burden.

\section{Study Population and protocol}

Eligible subjects were consecutive male or females $>18$ years of age with an indication for permanent pacemaker therapy. Inclusion criteria were as follows: 1 . Normal left ventricular ejection fraction $>55 \% .2$. Normal to mildly dilated left ventricular end diastolic diameter $<57 \mathrm{~mm}$. 3. Normal right ventricular function TAPSE $>17 \mathrm{~mm}$. If a patient fulfilled all inclusion criteria, clinical data and echocardiographic parameter were analysed prospectively at the time of pacemaker implantation and after 6 and 12 month. Additionally, for long time follow-up of the left ventricular function, in further 50 patients a retrospective analysis of the left and right ventricular function was performed at the time of pacemaker implantation and after 24 month.

\section{Pacemaker implantation, programming and follow-up}

The type of pacemaker to be implanted was selected by the responsible physician according to bradyarrhythmia type and the current guidelines. Correct positioning of implanted leads in the right ventricular septal apex was confirmed by fluoroscopy in RAO and LAO view. The pacemakers were programmed immediately after implantation according to the pace maker indication and the guidelines.

The first pacemaker follow-up was performed 4 weeks postoperatively. Further monitoring was performed after 6 and 12 months including the registration of the RV apical pacing burden.

\section{Transthoracic echocardiography}

In all subjects an echocardiogram was performed to assess LVEDD, LVESD, LVEF, E/A-ratio; E/E'-ratio and TAPSE at the time of enrollment, which was repeated at 6 and 12 months after pacemaker implantation. In the retrospective study group, LVEF and TAPSE were analyzed at the time of pacemaker implantation and 24 month afterwards. For the echocardiographic examinations, the iE33 xMatrix-DS ultrasound device from Philips (Koninklijke Philips N. V., Amsterdam, The Netherlands), with a $2.5 \mathrm{MHz}$ transducer was used. All acquired echocardiograms were reviewed by a core laboratory at the Heart Center Dresden. The LVEDD and LVESD was measured M-mode guided in the parasternal long-axis view. The LVEF was assessed by biplane Simpson's rule using manual tracing of digital images. ${ }^{5}$ TAPSE was determined with M-mode guiding in the apical 4-chambers view. Thereby, the maximal systolic excursion of the RV free 
wall at the junction with the tricuspid valve was measured. ${ }^{6}$ The evaluation of LV diastolic function was determined, measuring the mitral inflow and DTI of the mitral lateral annulus. The E and A peaks as well as the $e^{\prime}$ and $a^{\prime}$ peaks were measured, followed by the calculation of the E/A-ratio and E/E'-ratio. ${ }^{7}$

\section{Spiroergometry}

In all subjects a spiroergometry was performed to assess maximal oxygen consumption $\left(\mathrm{VO}_{2} \mathrm{max}\right)$, peak exercise capacity and ventilatory anaerobic threshold (VAT) at the time of enrollment, which was repeated at 6 and 12 months after pacemaker implantation.

After resting for 5 minutes, the patients were examined, sitting on the bicycle ergometer. During exercise, 12 ECG leads were recorded and the heart rate was continuously calculated with the help of R-R interval. The arterial blood pressure was measured at rest and at least one time per resistance level. Each patient underwent exercise testing using a continuously increasing resistance of $25 \mathrm{~W}$ every 2 minutes, starting at $0 \mathrm{~W}$ (ramp protocol). Subjects were exercised to their self-determined maximal capacity or until the test was stopped because of one of the following criteria: chest pain or dizziness of the patient, potentially dangerous arrhythmias or ST-segment deviations, marked systolic hypotension or hypertension. ${ }^{8}$ During the test, the patient breathed through a tight-fitting mask. Thereby, the inhaled and the exhaled air volume and flow were measured by a connected spirometer. Additionally, the oxygen consumption $\left(\mathrm{VO}_{2}\right)$ and carbon dioxide production $\left(\mathrm{VCO}_{2}\right)$ were quantified. Maximal oxygen consumption $\left(\mathrm{VO}_{2} \mathrm{max}\right)$ was defined as the highest $\mathrm{VO}_{2}$ obtained during the entire test and is expressed in $\mathrm{ml} / \mathrm{min} / \mathrm{kg} .{ }^{9}$ The ventilator anaerobic threshold VAT was determined as the first non-linear increase in the ventilatory equivalent for oxygen without a simultaneous increase of the ventilatory equivalent for $\mathrm{CO}_{2} \cdot{ }^{10}$

\section{Statistical analysis}

Data were tested for normal distribution. Results of continuous variables are expressed as means \pm standard deviation. Statistical analyses were done using the 2 tailed, unpaired Student's t-test. Level of significance was set to $p<0.05$. Categorical variables are presented as total number with comparison using chi-square statistics and Fisher exact test. If more than 2 groups were analyzed, a one-way ANOVA test was performed. Post hoc analyses have been applied using Bonferroni method. Significance level was set to $p<0.05$.

\section{Results}

\section{Study population and procedural data:}

In total 60 consecutive patients were included in the current prospective analyses. In addition 48 patients were included retrospectively for long time follow-up. Thereby, the patients were divided into two groups: The high RV pacing burden group (hRVP) was defined as subjects with a RV pacing $\geq 40 \%$ according to the definitions in previous randomized trials. ${ }^{3}$ In contrast, the low RV pacing burden group (IRVP) was defined as RV pacing $<40 \%$. The demographics and clinical baseline characteristics are shown in Table 1. 
Both treatment groups were well balanced in regards to the demographics and clinical baseline characteristics. Of note the patients in the hRVP group suffered significantly more from AV block (100\%) in comparison to the IRVPgroup (25.6\%). In the retrospective cohort the patients in the hRVP group were significantly older compared to the IRVP subjects (58 years vs. 72 years; $p<0.05$ ). Additionally, in the retrospective cohort singnificantly more 2 chamber pacemaker were implanted in the IRVP patients. There were no relevant differences in gender, co-morbidities and concomitant medications in both groups. 
Table 1

baseline characteristics

\begin{tabular}{|c|c|c|c|c|}
\hline & RV-pacing & IRVP & hRVP & p-value \\
\hline \multirow[t]{18}{*}{ (prospective) } & Total population & 39 & 21 & \\
\hline & Age, year & $71(11.4)$ & $72(12.4)$ & 0.6 \\
\hline & Sex, male N (\%) & $21(53.8)$ & $14(66.7)$ & 0.4 \\
\hline & Comorbidities & & & \\
\hline & Hypertension N (\%) & $34(87.2)$ & $18(85.7)$ & 1.0 \\
\hline & Diabetes mellitus N (\%) & $22(56.4)$ & $14(66.7)$ & 0.58 \\
\hline & CAD N (\%) & $24(63.2)$ & $15(71.4)$ & 0.57 \\
\hline & A Fib N (\%) & $5(12.8)$ & $7(33.3)$ & 0.09 \\
\hline & Medications & & & \\
\hline & Beta blocker N (\%) & $34(87.2)$ & $18(85.7)$ & 1.0 \\
\hline & ACE-inhibitor N (\%) & $34(87.2)$ & $34(87.2)$ & 1.0 \\
\hline & Pacemaker- Indications & & & \\
\hline & SSS N (\%) & $29(74.4)$ & $0(0)$ & $<0.05$ \\
\hline & AV-Block N (\%) & $10(25.6)$ & $21(100)$ & \\
\hline & Type of Pacemakers & & & \\
\hline & Single chamber $\mathrm{N}(\%)$ & $2(5.1 \%)$ & $5(23.8 \%)$ & 0.25 \\
\hline & Dual-chamber N (\%) & $37(94.9)$ & $16(76.2 \%)$ & \\
\hline & RV-pacing & IRVP & hRVP & $\mathrm{p}$-value \\
\hline \multirow[t]{8}{*}{ (retrospective) } & Total population & 31 & 17 & \\
\hline & Age, year & $58(13.5)$ & $72(6.1)$ & $<0.05$ \\
\hline & Sex, male N (\%) & $19(61.3)$ & $8(47.1)$ & 0.37 \\
\hline & Comorbidities & & & \\
\hline & Hypertension N (\%) & $26(83.9)$ & $16(94.1)$ & 0.40 \\
\hline & Diabetes mellitus N (\%) & $10(32.3)$ & $5(29.4)$ & 1.0 \\
\hline & CAD N (\%) & $19(61.3)$ & $14(82.4)$ & 0.196 \\
\hline & A Fib N (\%) & $1(3.2)$ & $7(41.2)$ & $<0.05$ \\
\hline
\end{tabular}




\begin{tabular}{|c|c|c|c|}
\hline RV-pacing & IRVP & hRVP & p-value \\
\hline \multicolumn{4}{|l|}{ Medications } \\
\hline Beta blocker N (\%) & $26(83.9)$ & $17(100)$ & 0.14 \\
\hline ACE-inhibitor N (\%) & $26(83.9)$ & $16(94.1)$ & 0.40 \\
\hline \multicolumn{4}{|l|}{ Pacemaker- Indications } \\
\hline SSS N (\%) & $19(61.3)$ & $1(5.9)$ & $<0.05$ \\
\hline AV-Block N (\%) & $3(9.7)$ & $15(88.2)$ & \\
\hline Unknown N (\%) & $9(29)$ & $1(5.9)$ & \\
\hline \multicolumn{4}{|l|}{ Type of Pacemakers } \\
\hline Single chamber N (\%) & $0(0)$ & $5(29.4)$ & $<0.05$ \\
\hline Dual-chamber N (\%) & $31(100)$ & $12(70.6)$ & \\
\hline \multicolumn{4}{|l|}{ IRVP: RV pacing < 40\% ; hRVP: RV pacing $\geq 40 \%$} \\
\hline \multicolumn{4}{|c|}{$\begin{array}{l}\text { CAD: coronary artery disease; A Fib: atrial fibrillation; SSS: sick sinus syndrome; AV-Block: } \\
\text { atrioventricular Block; ACE-Inhibitor: angiotensin-converting enzyme }\end{array}$} \\
\hline
\end{tabular}

\section{Change of echocardiographic parameter in dependence of the RV pacing burden (primary endpoint)}

The development of the echocardiographic parameters over the course of one year in dependence of the $\mathrm{RV}$ pacing burden is shown in Fig. 1 for the prospective cohort and in Fig. 2 for the long time follow-up in the retrospective cohort. In short, none of the measured echocardiographic parameter changed significantly in the time course of 6,12 and 24 month, independently of the burden of RV pacing (LVEDD. IRVP: baseline: $45.4 \pm 0.64 \mathrm{~mm}, 6$ month: $45.5 \pm 0.58 \mathrm{~mm}, 12$ month: $46.4 \pm 0.51 \mathrm{~mm}$, hRVP: baseline: $46.4 \pm 1.4 \mathrm{~mm}, 6$ month: $46.5 \pm 1.1 \mathrm{~mm}, 12$ month: $47.2 \pm 1.0 \mathrm{~mm}$; LVESD: IRVP: baseline: $32.2 \pm 0.74 \mathrm{~mm}$, 6 month: $32.6 \pm 0.62 \mathrm{~mm}, 12$ month: $32.8 \pm 0.61 \mathrm{~mm}$, hRVP: baseline: $31.7 \pm 1.5 \mathrm{~mm}, 6$ month: $32.6 \pm 1.2$ mm, 12 month: $32.4 \pm 1.1 \mathrm{~mm}$; LVEF: IRVP: baseline: $59.2 \pm 1.0 \%, 6$ month: $57.9 \pm 0.93 \%, 12$ month: 57.6 $\pm 0.77 \%$, 24 month: $56.7 \pm 0.99 \%$; hRVP: baseline: $59.7 \pm 1.6 \%, 6$ month: $58.5 \pm 1.1 \%, 12$ month: $58.8 \pm$ $0.9 \%, 24$ month: $55.4 \pm 2.0 \%$; TAPSE: IRVP: baseline: $23.7 \pm 0.71 \mathrm{~mm}, 6$ month: $23.5 \pm 0.76 \mathrm{~mm}, 12$ month: $23.4 \pm 0.57 \mathrm{~mm}, 24$ month: $24.0 \pm 0.34 \mathrm{~mm}$ hRVP: baseline: $26.0 \pm 0.85 \mathrm{~mm}, 6$ month: $24.2 \pm 0.76$ mm, 12 month: $23.3 \pm 0.58 \mathrm{~mm}, 24$ month: $23.2 \pm 0.66 \mathrm{~mm}$; E/Aratio: IRVP: baseline: $1.0 \pm 0.05,6$ month: $1.05 \pm 0.05,12$ month: $0.98 \pm 0.04$, hRVP: baseline: $1.1 \pm 0.07,6$ month: $1.1 \pm 0.06,12$ month: $1.19 \pm 0.08$; E/E'-ratio: IRVP: baseline: $9.3 \pm 0.5,6$ month: $8.6 \pm 0.44,12$ month: $8.0 \pm 0.31$, hRVP: baseline: $10.2 \pm 1.0,6$ month: $9.5 \pm 0.77,12$ month: $8.8 \pm 0.59)$. 


\section{Change of spiroergometric parameter in dependence of the $R V$ pacing burden (clinical endpoint)}

Figure 3 shows the development of the spiroergometric parameters over the course of one year in dependence of the RV pacing burden. Analogous to the echocardiographic data, no relevant changes of spiroergometric parameters could be detected in the period of 12 month regardless of the RV pacing burden

(peak exercise capacity. IRVP: baseline: $105 \pm 7$ W, 6 month: $115 \pm 6$ W, 12 month: $114 \pm 7$ W, hRVP: baseline: $114 \pm 12 \mathrm{~W}, 6$ month: $118 \pm 14 \mathrm{~W}, 12$ month: $127 \pm 15 \mathrm{~W}$; $V_{2} \max$. IRVP: baseline: $22.5 \pm 0.8$ $\mathrm{ml} / \mathrm{min} / \mathrm{kg}, 6$ month: $22.8 \pm 0.8 \mathrm{ml} / \mathrm{min} / \mathrm{kg}, 12$ month: $23.1 \pm 24.4 \mathrm{ml} / \mathrm{min} / \mathrm{kg}$, hRVP: baseline: $21.4 \pm 1.4$ $\mathrm{ml} / \mathrm{min} / \mathrm{kg}, 6$ month: $22.9 \pm 1.9 \mathrm{ml} / \mathrm{min} / \mathrm{kg}, 12$ month: $24.4 \pm 2.0 \mathrm{ml} / \mathrm{min} / \mathrm{kg} ; V A T$ : IRVP: baseline: $89 \pm 6$ W, 6 month: $86 \pm 6$ W, 12 month: $86.2 \pm 6$ W, hRVP: baseline: $82 \pm 14$ W, 6 month: $81 \pm 14$ W, 12 month: 85 $\pm 14 \mathrm{~W})$

\section{Discussion}

The present prospective pacemaker registry is one of the most detailed studies regarding the influence of RV pacing on various echocardiographic and spiroergometric parameters. As in numerous previous pacemaker studies, this trial could not reveal any relevant short term changes of several echocardiographic and spiroergometric parameter regardless to the burden of RV pacing in patients with preserved ejection fraction. Several clinical studies have reported that chronic right ventricular (RV) apical pacing causes adverse cardiovascular outcomes, including negative cardiac remodeling, atrial fibrillation (AF), congestive heart failure (HF), and mortality. ${ }^{3,11,12}$ Moreover, it was shown that adverse left ventricular remodelling caused by conventional right ventricular apical pacing, could be prevented by biventricular pacing. ${ }^{12}$ Based on the present detailed echocardiographic data, it is difficult to explain the deteriorating clinical outcome of RV pacing in patients with preserved left ventricular ejection fraction. A large cohort study of pacemaker recipients with predominantly normal LVEF, was also unable to demonstrate a clinically relevant decrease in LVEF due to RV pacing even after 44 months of follow-up. ${ }^{4}$ While this study focused solely on the systolic left ventricular function, the current trial also examined the diastolic left ventricular function over a period of 12 month as well as the right ventricular function after 24 month of follow-up. But also the analysis of these echocardiographic parameters consistently showed the same result: The burden of RV apical pacing has no influence on the left and right systolic and diastolic function in patients with preserved LVEF.

These echocardiographic results were also reflected in the primary clinical short term outcome of the current trial, which was determined by spiroergometry. Accordingly to the echo data no relevant changes of peak exercise capacity, ventilatory anaerobic threshold and maximal oxygen consumption could be revealed independently of the RV pacing burden in the short term follow-up. As this is the first pacemaker study, which defines the clinical outcome in spiroergometric parameters, no direct comparison with 
previous trials, determining clinical outcome as congestive heart failure, atrial fibrillation or mortality, is possible.

However, few data exist, showing that isn't the RV pacing burden itself but the programmed AV interval which influence the exercise capacity. ${ }^{13}$ Therefore, if a patient's exercise capacity deteriorate under RV pacing, as primary noninvasive approach an echocardiographic optimisation of AV interval should be considered to improve the exercise capacity.

A very important aspect regarding the adverse potential of RV pacing seems to be the initial LV function. Thus in the DAVID trial patients with standard indications for ICD therapy and an LVEF of $40 \%$ or less, RV pacing increased the combined end point of death or hospitalization for heart failure. ${ }^{3}$ Furthermore, the Mode selection trial (MOST) could reveal an increased risk of heart failure and atrial fibrillation over a period of 6 years in patients with a cumulative RV pacing $>40 \%$ compared to patients with a low RV pacing burden. ${ }^{2}$ Of note, in the MOST trial the clinical endpoints were not analysed with respect of the LV function. Also, the BLOCK-HF study suggests that patients with LVEF $<50 \%$ and AV block may benefit from biventricular pacing in terms to heart failure compared to RV pacing. ${ }^{14}$

In recent years, due to the ongoing discussion about the adverse potential of RV stimulation, various pacemaker programming algorithms, switching from $\operatorname{AAI}(R)$ to $\operatorname{DDD}(R)$, a have been developed to reduce unnecessary RV stimulation as much as possible. However, the subsequent MVP trial (Managed Ventricular pacing vs.VVI 40 Pacing) failed to demonstrate non-inferiority of this algorithm. ${ }^{15}$ Also a previous meta-analysis could demonstrate that the reduction of unnecessary ventricular pacing fails to affect hard clinical outcomes in patients with preserved left ventricular function. ${ }^{16}$ In addition, it could be shown, that the burden of RV pacing does not influence the quality of life in pace maker patients. ${ }^{17}$

Inspired by an excellent editorial, written by Dr. Israel about his "top 10 excuses" with regard to unexpected results in prospective pacemaker studies, this study has several limitations as well. ${ }^{18}$ First, the current study subjects were not randomised into the high and low RV pacing group. Furthermore, the study population was too small to make valid conclusions about clinical endpoints like heart failure or mortality. Additionally, the current study cannot rule out long-term negative effects of RV stimulation, as the follow-up period did not exceed 24 months.

In summary of all clinical studies as well as existing and current echocardiography/spiroergometry studies, the mechanism of worsened pacemaker-induced clinical outcome remains unclear, especially in patients with preserved left ventricular function. Therefore, further studies are needed to reveal which patients with preserved left ventricular function benefit clinically from HIS bundle or left ventricular pacing.

\section{Conclusions}


In pacemaker patients with preserved LVEF the burden of RV pacing has no adverse influence neither to several echocardiographic parameters nor to the clinical exercise capacity after a follow-up of 12 to 24 month. Therefore, the mechanism of the worsened clinical outcome due to high RV pacing burden in patients without a relevant structural heart disease remains unclear.

\section{Declarations}

\section{Acknowledgements:}

We thank all staff of the cardiopulmonary function diagnostics, Heart Center Dresden for supporting this registry

\section{Funding:}

This study was not supported by any grant.

\section{Conflicts of interest/Competing interests:}

All authors report no conflicts of interests

\section{Availability of data and material (data transparency)}

The data that support the findings of this study are available from the first author, AY, upon reasonable request.

\section{Ethics approval:}

The study was approved by the institutional ethical review board. All data were collected, managed and analysed at the Heart Centre, University of Dresden (ethics votum University of Dresden: EK 28409202).

\section{Patient consent statement:}

Informed consent was obtained from all individual participants included in the study.

\section{References}

1. Sharma AD, Rizo-Patron C, Hallstrom AP, O'Neill GP, Rothbart S, Martins JB, Roelke M, et al. Percent right ventricular pacing predicts outcomes in the DAVID trial. Heart Rhythm 2005;2:830-4.

2. Sweeney MO, Hellkamp AS, Ellenbogen KA, Greenspon AJ, Freedman RA, Lee KL, Lamas GA. Adverse effect of ventricular pacing on heart failure and atrial fibrillation among patients with normal baseline QRS duration in a clinical trial of pacemaker therapy for sinus node dysfunction. Circulation 2003;107:2932-7.

3. Wilkoff BL, Cook JR, Epstein AE, Greene HL, Hallstrom AP, Hsia H, Kutalek SP, et al. Dual-chamber pacing or ventricular backup pacing in patients with an implantable defibrillator: the Dual Chamber 
and VVI Implantable Defibrillator (DAVID) Trial. JAMA 2002;288:3115-23.

4. Ebert M, Jander N, Minners J, Blum T, Doering M, Bollmann A, Hindricks G, et al. Long-Term Impact of Right Ventricular Pacing on Left Ventricular Systolic Function in Pacemaker Recipients With Preserved Ejection Fraction: Results From a Large Single-Center Registry. J Am Heart Assoc 2016;5.

5. Shah AM, Claggett B, Sweitzer NK, Shah SJ, Anand IS, Liu L, Pitt B, et al. Prognostic Importance of Impaired Systolic Function in Heart Failure With Preserved Ejection Fraction and the Impact of Spironolactone. Circulation 2015;132:402 - 14.

6. Kaul S, Tei C, Hopkins JM, Shah PM. Assessment of right ventricular function using two-dimensional echocardiography. Am Heart J 1984;107:526 - 31.

7. Nagueh SF, Smiseth OA, Appleton CP, Byrd BF 3rd, Dokainish H, Edvardsen T, Flachskampf FA, et al. Recommendations for the Evaluation of Left Ventricular Diastolic Function by Echocardiography: An Update from the American Society of Echocardiography and the European Association of Cardiovascular Imaging. Eur Heart J Cardiovasc Imaging 2016;17:1321-60.

8. Duncan GE, Howley ET, Johnson BN. Applicability of VO2max criteria: discontinuous versus continuous protocols. Med Sci Sports Exerc 1997;29:273-8.

9. Arena R, Humphrey R, Peberdy MA. Prognostic ability of VE/VCO2 slope calculations using different exercise test time intervals in subjects with heart failure. Eur J Cardiovasc Prev Rehabil 2003;10:4638.

10. Wasserman K, Beaver WL, Whipp BJ. Gas exchange theory and the lactic acidosis (anaerobic) threshold. Circulation 1990;81:II14-30.

11. Sweeney MO, Bank AJ, Nsah E, Koullick M, Zeng QC, Hettrick D, Sheldon T, et al. Minimizing ventricular pacing to reduce atrial fibrillation in sinus-node disease. N Engl J Med 2007;357:1000-8.

12. Yu CM, Chan JY, Zhang Q, Omar R, Yip GW, Hussin A, Fang F, et al. Biventricular pacing in patients with bradycardia and normal ejection fraction. N Engl J Med 2009;361:2123-34.

13. Khairy P, Talajic M, Dominguez M, Tardif JC, Juneau M, Lavoie L, Roy D, et al. Atrioventricular interval optimization and exercise tolerance. Pacing Clin Electrophysiol 2001;24:1534-40.

14. Curtis AB, Worley SJ, Adamson PB, Chung ES, Niazi I, Sherfesee L, Shinn T, et al. Biventricular pacing for atrioventricular block and systolic dysfunction. N Engl J Med 2013;368:1585-93.

15. Sweeney MO, Ellenbogen KA, Tang AS, Whellan D, Mortensen PT, Giraldi F, Sandler DA, et al. Atrial pacing or ventricular backup-only pacing in implantable cardioverter-defibrillator patients. Heart Rhythm 2010;7:1552-60.

16. Shurrab M, Healey JS, Haj-Yahia S, Kaoutskaia A, Boriani G, Carrizo A, Botto G, et al. Reduction in unnecessary ventricular pacing fails to affect hard clinical outcomes in patients with preserved left ventricular function: a meta-analysis. Europace 2017;19:282 - 88.

17. Maurer DE, Naegeli B, Straumann E, Bertel O, Frielingsdorf J. Quality of life and exercise capacity in patients with prolonged $\mathrm{PQ}$ interval and dual chamber pacemakers: a randomized comparison of permanent ventricular stimulation vs intrinsic AV conduction. Europace 2003;5:411-7. 
18. Israel CW. Pacing-induced heart failure: should we avoid right ventricular pacing or not? EP Europace 2017;19:165 - 68.

\section{Figures}

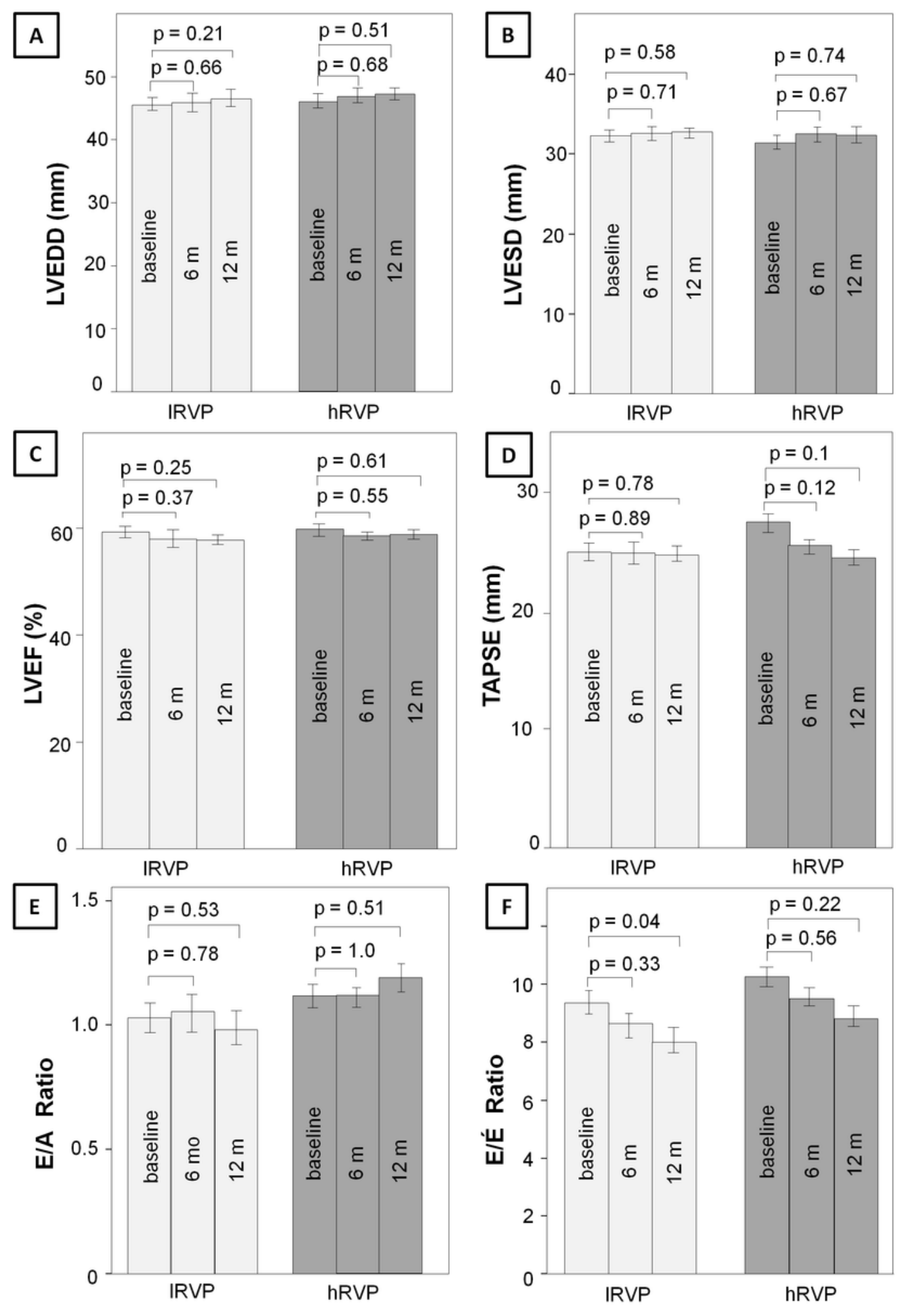

Figure 1 
Time course of echocardiographic parameters during RV pacing (prospective analyses) IRVP: RV pacing $<40 \%$; hRVP: RV pacing $\geq 40 \% ; 6$ m: 6 month; 12 m: 12 month; A: LVEDD left ventricular end diastolic diameter in mm; B: LVESD left ventricular end systolic diameter in $\mathrm{mm}$; C: LVEF: left ventricular ejection fraction in \%; D: TAPSE: Tricuspid Annular Plane Systolic Excursion; E: diastolic function E/A Ratio; F: diastolic function E/E' Ratio
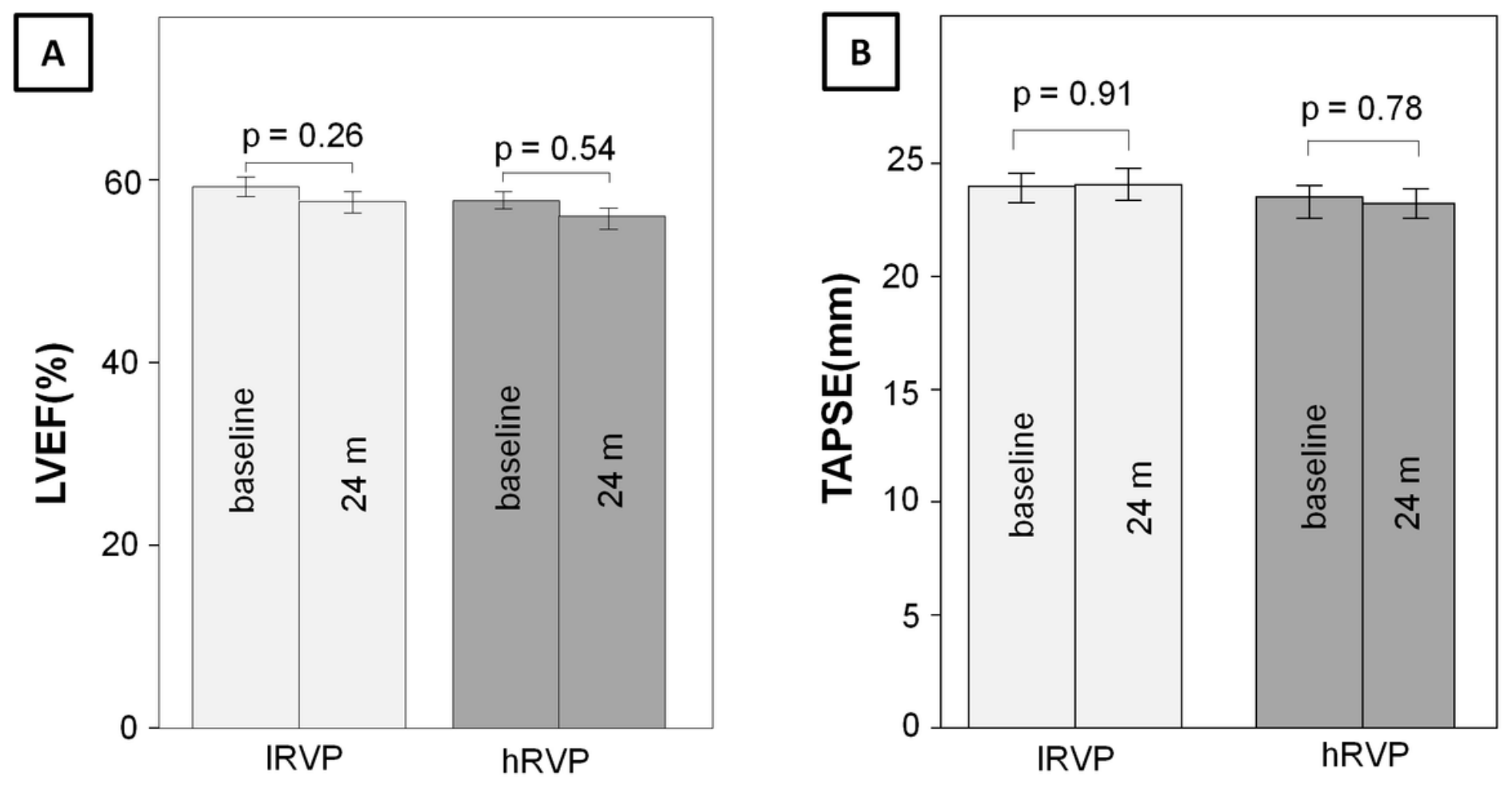

Figure 2

Time course of echocardiographic parameters during RV pacing (retrospective analyses) IRVP: RV pacing $<40 \%$; hRVP: RV pacing $\geq 40 \%$; 24 m: 24 month; A: LVEF: left ventricular ejection fraction in \%; B: TAPSE: Tricuspid Annular Plane Systolic Excursion 


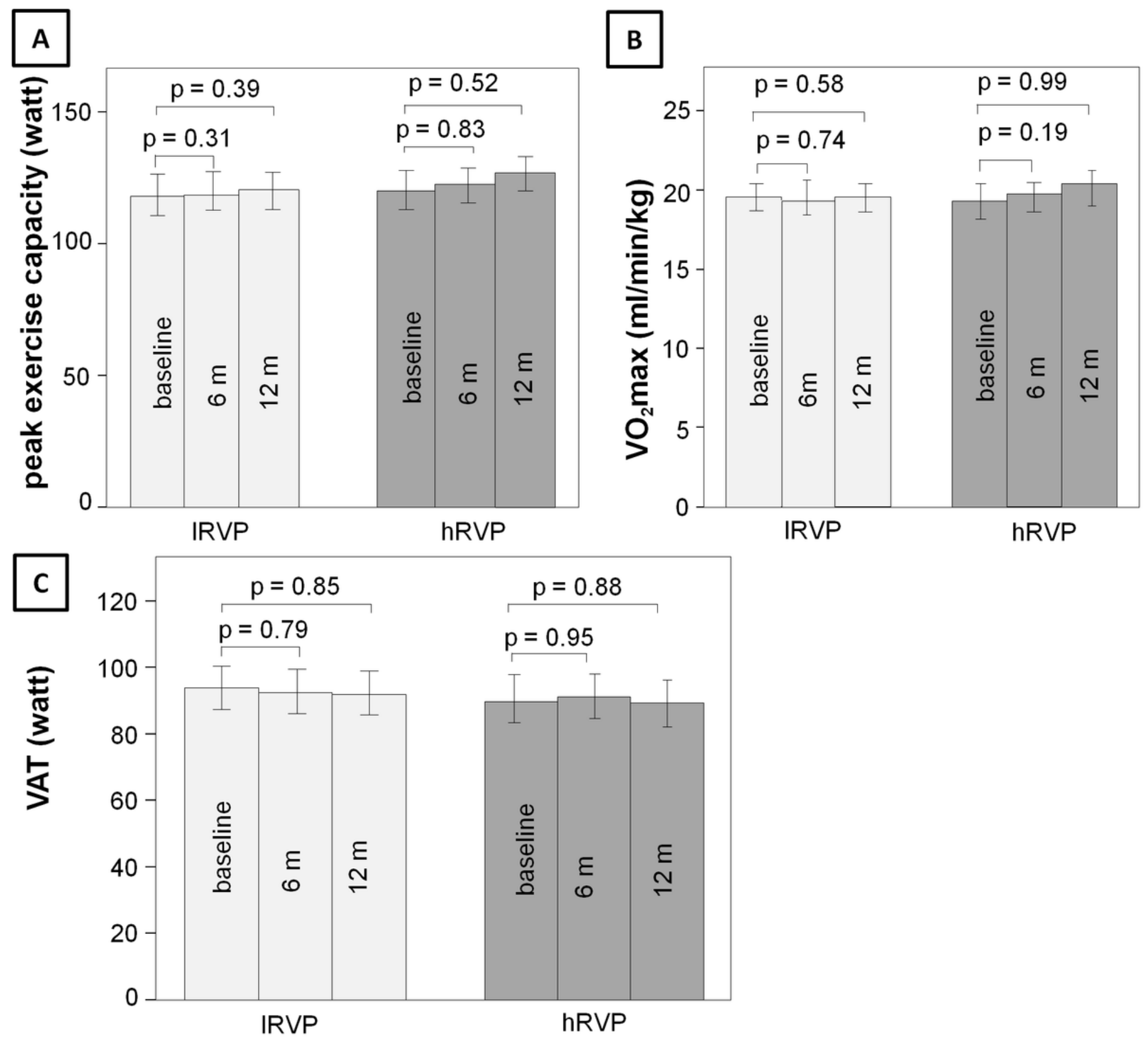

Figure 3

Time course of spiroergometric parameters during RV pacing (prospective analyses) IRVP: RV pacing < 40\%; hRVP: RV pacing $\geq 40 \% ; 6$ m: 6 month; 12 m: 12 month; A: peak exercise capacity in watt; B: VO2max: maximal oxygen consumption in $\mathrm{ml} / \mathrm{min} / \mathrm{kg}$; C: VAT: ventilator anaerobic threshold in watt 